\title{
Electrolytes Alterations in Some Organs due to Lead Exposure
}

\section{Okediran B Samuel*, Sanusi F and Suleiman K Yusuf \\ Department of Veterinary Physiology and Biochemistry, University of Ilorin, Nigeria}

*Corresponding author: Okediran B Samuel, Department of Veterinary Physiology and Biochemistry, University of Ilorin, Ilorin, Nigeria, Tel: +2348034241610; Email: okediranbabatunde@gmail.com

\section{Research Article \\ Volume 4 Issue 1}

Received Date: March 13, 2019

Published Date: March 29, 2019

DOI: $10.23880 /$ act- 16000150

\section{Abstract}

Background: Lead is one of the metals whose toxicological effect in humans and animals is of clinical concerned. Once it is absorbed, it is distributed via the blood to various tissues and organs where they are stored. Electrolytes played significant role in metabolic and physiological processes taken in the body. We investigated some electrolytes distribution in the blood, liver, kidney, spleen and brain of male Albino rats exposed to different concentrations of lead as lead acetate.

Methods: A total of sixteen male Wistar rats were used for the experiment. They were randomly divided into four groups (A-D) of four animals per group and were treated with lead acetate orally as the source of lead exposure for a period of four, eight and twelve weeks.

Results: The results showed a significant increase in blood lead concentration and decrease in plasma sodium, potassium, and calcium and magnesium concentrations. The electrolytes concentrations in various organs studied showed different patterns of variations which were dose and time dependent.

Conclusions: It can be concluded that exposure to lead could result in perturbation of electrolytes in blood and different organs thus interfering with electrolytes metabolism resulting in deterioration of cellular functions.

Keywords: Lead; Electrolytes; Blood; Liver; Kidney

\section{Introduction}

Lead is a poisonous metal, which exist in both organic (Tetraethyl lead) and inorganic (lead acetate, lead chloride) forms in the environment [1]. It has been used in medicines, paintings, pipes, ammunition and in more recent times in alloys for welding storage materials for chemical reagents [2]. Exposure to lead is from numerous sources and a myriad of pathways including air, food, dust, soil and water [3]. Lead has very high affinity for red blood cells, it has been shown that lead inhibits the enzymes-aminolevulinic acid dehydratase (ALAD) and ferrochelatase of the hem synthetic pathway thus preventing conversion of ALA to porphobilinogen and inhibits incorporation of iron into the protoporphyrin ring respectively. This results in reduced hem synthesis and elevated levels of the precursor-aminolevulinic acid (ALA), which is a weak gamma-amino butyric acid (GABA) agonist that decreases GABA release by presynaptic inhibition [4,5]. Electrolytes are present in body tissues and body fluids and are necessary for the maintenance of physiochemical conditions like secretion of hydrochloric acid in stomach, blood coagulation, bone matrix, membrane potential, bioluminescence and blood pressure 


\section{Advances in Clinical Toxicology}

which are essential for life. Electrolytes are structural components of body soft tissues like liver, muscle and also participate in acid-base balance and are components of blood buffers.

Approximately $90 \%$ of absorbed lead is reported to be stored in the bone with a half-life of 600 - 3000 days. The remaining $10 \%$ is stored in soft tissues like kidney, liver and brain. The half-life of lead in these tissues ranges from 40 - 50 days [6]. Lead is conjugated in the liver and passed to the kidney, where a small quantity is excreted in urine and the rest accumulates in various body organs, affecting many biological activities at the molecular, cellular and intercellular levels, which may result in morphological alterations that can remain even after lead levels have fallen [7-9]. Lead toxicity is closely related to it accumulation in various tissues and it interference with the electrolytes that hamper several physiological processes [10].

Hence, this present study is to determine some electrolytes such as sodium, potassium, calcium and magnesium in some organs such as liver, kidney, spleen and brain of male Albino rats treated with graded doses of lead for a period of four, eight and twelve weeks.

\section{Materials and methods}

\section{Experimental Animals}

A total of sixteen male Wistar rats were used for this investigation. The average weight of the rats was $152 \pm 3.50 \mathrm{~g}$. They were provided with laboratory animal feed (Fat/oil 6\%, crude fibre 5\%, calcium 1\%, Available phosphorus $0.4 \%$, Lysine $0.85 \%$, Methionine $0.35 \%$, Salt $0.3 \%$, Crude protein 18\%, Metabolisable Energy 2900 Kcal.kg-1, manufactured by TOPFEEDS $囚$, (Lagos, Nigeria) and water provided. Experimental animals were acclimatized to their environment before the start of the experiment.

\section{Animal Ethics}

All experimental protocols carried out on the animals were in accordance with the international accepted principles for laboratory animal use and were approved by the Ethics Committee (UIL/FVERC/001/2018) on Laboratory animal use of the Faculty of Veterinary Medicine, University of Ilorin, Nigeria.

\section{Chemical Exposure}

A total of sixteen male Wistar rats were used for the experiment. They were randomly divided into four groups (A-D) of four animals per group and were treated with lead acetate orally as the source of lead exposure for a period of four, eight and twelve weeks. The treatments were as follows:

Group A was the control non exposed group while groups B to D were exposed to $200 \mathrm{ppm}, 300 \mathrm{ppm}$ and $400 \mathrm{ppm}$ of lead as lead acetate respectively.

Sample collections: Blood samples were collected into heparinised tubes by cardiac puncture after an overnight fast. The animals were euthanized after which the following organs liver, kidney, spleen and brain were finely excised for biochemical analyses.

Blood lead determination: Blood lead concentration were determined in whole blood using atomic absorption spectrophotometer (Buck 200) a product of Buck scientific, Connecticut, U.S.A.

Preparation of blood plasma: The blood samples were centrifuged at $4000 \mathrm{rpm}$ for 10 minutes to separate the plasma from the red blood cells. The plasma was then removed and stored in Eppendorf tubes for further analyses.

Isolation of organs: The following organs were harvested after the rats were euthanized liver, kidney spleen and brain. These organs were finely excised and washed with $20 \mathrm{mM}$ Tris buffer $\mathrm{pH}$ 7.6.

Extraction of electrolytes from organs: Extraction of electrolytes from organs followed the procedure of Johny et al. [11]. 1.0g of the organ was homogenized in $10 \mathrm{ml}$ of $20 \mathrm{mM}$ Tris buffer $\mathrm{pH}$ 7.6. This was then centrifuged at $5000 \mathrm{rpm}$ for 10 minutes. The supernatant was decanted and kept in Eppendorff tubes for analysis.

Biochemical analyses: Plasma sodium, potassium, calcium and magnesium concentrations were determined spectrophotometrically according to the method of Trinder, [12], Terri and Sesin [13], Schmidt-Gayk, et al. [14] and Abernethy and Fowler [15] as described in Cromatest ${ }^{\circledR}$ diagnostic kit manual respectively; while electrolytes in organs were determined by the modifications of the procedures for determination of electrolytes in plasma.

Statistical analysis: Results were expressed as mean \pm Standard Error of mean (SEM). Analysis of data was done 
using one way analysis of variance (ANOVA), followed by the Duncan multiple range test. A p value $<0.05$ was considered significant in all cases.

\section{Results}

The mean blood lead concentrations of male Wistar rats treated with graded doses of lead for a period of four, eight and twelve weeks are shown in Table 1. There was a significant increase $(\mathrm{p}<0.05)$ in blood lead concentrations in the treated groups compared to the control group. The 400ppm treated group had the highest blood lead concentration irrespective of the duration of lead treatment. The group treated with 400ppm for four weeks had blood lead concentration of $19.76 \pm 0.47 \mu \mathrm{g} / \mathrm{dl}$ which was about 9.2 times higher than that of the control, while those treated for eight weeks had blood lead concentration of $39.82 \pm 1.14 \mu \mathrm{g} / \mathrm{dl}$ and those treated for twelve weeks had blood lead concentration of $63.50 \pm$ $1.65 \mu \mathrm{g} / \mathrm{dl}$ which was about 9.9 times higher than that of the control.

\begin{tabular}{|c|c|c|c|}
\hline & \multicolumn{3}{|c|}{ Blood lead concentration ( $\boldsymbol{\mu g} / \mathbf{d l})$} \\
\hline Groups (n=6) & 4 weeks & 8 weeks & $\mathbf{1 2}$ weeks \\
\hline Control & $2.15 \pm 0.06 \mathrm{a}$ & $4.31 \pm 0.14 \mathrm{a}$ & $6.41 \pm 0.24 \mathrm{a}$ \\
\hline $200 \mathrm{ppm}$ & $12.36 \pm 1.14 \mathrm{~b}$ & $28.35 \pm 1.57 \mathrm{~b}$ & $39.39 \pm 2.92 \mathrm{~b}$ \\
\hline $300 \mathrm{ppm}$ & $13.16 \pm 1.00 \mathrm{~b}$ & $29.64 \pm 1.86 \mathrm{~b}$ & $43.60 \pm 2.77 \mathrm{~b}$ \\
\hline $400 \mathrm{ppm}$ & $19.76 \pm 0.47 \mathrm{c}$ & $39.82 \pm 1.14 \mathrm{c}$ & $63.50 \pm 1.65 \mathrm{c}$ \\
\hline
\end{tabular}

Table 1: The Mean Blood Lead Concentrations of Male Wistar Rats Treated with Graded Doses of Lead for a Period of Four, Eight and Twelve Weeks.

Values are mean \pm SEM. Values within the same column with different superscripts are significantly different at $\mathrm{p}<0.05$

Table 2 shows the plasma sodium, potassium, calcium and magnesium concentrations in male Albino rats treated with graded doses of lead for a period of four, eight and twelve weeks. In the plasma, there was significant dose and period dependent decrease $(\mathrm{p}<0.05)$ in plasma sodium, potassium, calcium and magnesium concentrations in the treated groups compared to the control group throughout the duration of exposure to lead.

\begin{tabular}{|c|c|c|c|c|c|c|c|c|c|c|c|c|}
\hline \multirow[b]{2}{*}{$\begin{array}{l}\text { Group } \\
s(n=6)\end{array}$} & \multicolumn{3}{|c|}{$\begin{array}{c}\text { Plasma sodium } \\
\text { concentrations } \mathrm{mmol} / \mathrm{l}\end{array}$} & \multicolumn{3}{|c|}{$\begin{array}{c}\text { Plasma potassium } \\
\text { concentrations } \\
\text { mmol/l }\end{array}$} & \multicolumn{3}{|c|}{$\begin{array}{c}\text { Plasma calcium } \\
\text { concentrations } \\
\text { mmol/l }\end{array}$} & \multicolumn{3}{|c|}{$\begin{array}{c}\text { Plasma magnesium } \\
\text { concentrations } \\
\text { mmol/l }\end{array}$} \\
\hline & 4week & 8 week & 12week & $\begin{array}{c}\text { 4wee } \\
\mathrm{k}\end{array}$ & $\begin{array}{c}8 \text { wee } \\
k\end{array}$ & $\begin{array}{c}12 \text { wee } \\
\mathrm{k}\end{array}$ & $\begin{array}{c}4 \text { wee } \\
\mathrm{k}\end{array}$ & 8week & $\begin{array}{c}12 w e e \\
k\end{array}$ & $\begin{array}{c}4 w e e \\
\mathrm{k}\end{array}$ & $\begin{array}{c}8 w e e \\
k\end{array}$ & $\begin{array}{c}12 \mathrm{we} \\
\mathrm{ek}\end{array}$ \\
\hline $\begin{array}{c}\text { Contro } \\
1\end{array}$ & $\begin{array}{l}125.76 \\
\pm 4.97 \mathrm{~b}\end{array}$ & $\begin{array}{l}129.94 \\
\pm 8.97 \mathrm{~b}\end{array}$ & $\begin{array}{l}141.45 \\
\pm 2.28 \mathrm{~b}\end{array}$ & $\begin{array}{l}4.00 \pm \\
0.28 \mathrm{~b}\end{array}$ & $\begin{array}{l}4.28 \pm \\
0.31 \mathrm{~b}\end{array}$ & $\begin{array}{l}4.27 \pm \\
0.31 \mathrm{~b}\end{array}$ & $\begin{array}{l}0.91 \pm \\
0.10 \mathrm{~b}\end{array}$ & $\begin{array}{l}0.86 \pm \\
0.07 \mathrm{c}\end{array}$ & $\begin{array}{l}0.85 \pm \\
0.07 \mathrm{c}\end{array}$ & $\begin{array}{l}0.40 \pm \\
0.05 \mathrm{c}\end{array}$ & $\begin{array}{l}0.44 \pm \\
0.05 c\end{array}$ & $\begin{array}{l}0.42 \pm \\
0.10 \mathrm{~b}\end{array}$ \\
\hline $\begin{array}{l}200 \mathrm{pp} \\
\mathrm{m}\end{array}$ & $\begin{array}{c}87.33 \pm \\
5.05 \mathrm{a}\end{array}$ & $\begin{array}{c}110.32 \\
\pm 6.63 \mathrm{ab}\end{array}$ & $\begin{array}{l}101.27 \\
\pm 6.61 \mathrm{a}\end{array}$ & $\begin{array}{l}2.04 \pm \\
0.28 \mathrm{a}\end{array}$ & $\begin{array}{l}2.21 \pm \\
0.32 \mathrm{a}\end{array}$ & $\begin{array}{l}3.13 \pm \\
0.44 \mathrm{a}\end{array}$ & $\begin{array}{l}0.40 \pm \\
0.01 \mathrm{a}\end{array}$ & $\begin{array}{l}0.36 \pm \\
0.02 b\end{array}$ & $\begin{array}{c}0.47 \pm \\
0.10 \mathrm{a} \\
\mathrm{b}\end{array}$ & $\begin{array}{l}0.33 \pm \\
0.04 b\end{array}$ & $\begin{array}{l}0.29 \pm \\
0.04 \mathrm{~b}\end{array}$ & $\begin{array}{l}0.22 \pm \\
0.03 \mathrm{a}\end{array}$ \\
\hline $\begin{array}{l}300 \mathrm{pp} \\
\mathrm{m}\end{array}$ & $\begin{array}{l}85.18 \pm \\
4.78 \mathrm{a}\end{array}$ & $\begin{array}{l}102.18 \\
\pm 6.33 \mathrm{a}\end{array}$ & $\begin{array}{l}137.21 \\
\pm 6.48 \mathrm{~b}\end{array}$ & $\begin{array}{l}1.65 \pm \\
0.10 \mathrm{a}\end{array}$ & $\begin{array}{l}1.53 \pm \\
0.07 a\end{array}$ & $\begin{array}{c}3.65 \pm \\
0.19 \mathrm{a} \\
\mathrm{b}\end{array}$ & $\begin{array}{l}0.38 \pm \\
0.01 \mathrm{a}\end{array}$ & $\begin{array}{c}0.33 \pm \\
0.02 \mathrm{a} \\
\mathrm{b}\end{array}$ & $\begin{array}{l}0.59 \pm \\
0.11 b\end{array}$ & $\begin{array}{l}0.24 \pm \\
0.03 a\end{array}$ & $\begin{array}{l}0.17 \pm \\
0.01 \mathrm{a}\end{array}$ & $\begin{array}{l}0.13 \pm \\
0.02 \mathrm{a}\end{array}$ \\
\hline $\begin{array}{l}400 \mathrm{pp} \\
\mathrm{m}\end{array}$ & $\begin{array}{l}95.32 \pm \\
4.08 \mathrm{a}\end{array}$ & $\begin{array}{c}109.74 \\
\pm 7.07 \mathrm{ab}\end{array}$ & $\begin{array}{c}109.10 \\
\pm 10.94 \mathrm{a}\end{array}$ & $\begin{array}{l}1.84 \pm \\
0.30 \mathrm{a}\end{array}$ & $\begin{array}{l}2.03 \pm \\
0.33 \mathrm{a}\end{array}$ & $\begin{array}{c}3.63 \pm \\
0.10 \mathrm{a} \\
\mathrm{b}\end{array}$ & $\begin{array}{l}0.38 \pm \\
0.05 a\end{array}$ & $\begin{array}{l}0.25 \pm \\
0.02 \mathrm{a}\end{array}$ & $\begin{array}{l}0.26 \pm \\
0.03 \mathrm{a}\end{array}$ & $\begin{array}{l}0.18 \pm \\
0.03 a\end{array}$ & $\begin{array}{l}0.13 \pm \\
0.02 \mathrm{a}\end{array}$ & $\begin{array}{l}0.12 \pm \\
0.02 \mathrm{a}\end{array}$ \\
\hline
\end{tabular}

Table 2: The Mean Plasma Sodium, Potassium, Calcium and Magnesium Concentrations of Male Wistar Rats Treated with Graded Doses of Lead for A Period of Four, Eight and Twelve Weeks.

Values are mean \pm SEM. Values within the same column with different superscripts are significantly different at $\mathrm{p}<0.05$.

Table 3 shows the liver, kidney, spleen and brain sodium concentrations of male Wistar rats treated with graded doses of lead for a period of four, eight and twelve weeks. In the liver, there was significant increase in sodium concentration most especially in the groups exposed to 200ppm and 300ppm of lead, except at twelve weeks of treatment where there was significant $(\mathrm{p}<0.05)$ decrease. In the kidney, there was no significant change in the sodium concentration for the duration of four weeks of treatment, however there was significant $(\mathrm{p}<0.05)$ 
increase in kidney sodium concentration at 300ppm and $400 \mathrm{ppm}$ of lead for eight and twelve weeks of treatment. In the spleen there was significant decrease in the sodium concentration throughout the duration of treatment. In the brain, there was significant increase in sodium concentration throughout the duration of treatment. However the highest sodium concentration was observed in the group treated with $300 \mathrm{ppm}$ of lead for twelve weeks.

\begin{tabular}{|c|c|c|c|c|c|c|c|c|c|c|c|c|}
\hline & \multicolumn{3}{|c|}{$\begin{array}{c}\text { Liver sodium } \\
\text { concentrations } \mathbf{m g} / \mathbf{g}\end{array}$} & \multicolumn{3}{|c|}{$\begin{array}{c}\text { Kidney sodium } \\
\text { concentrations } \mathrm{mg} / \mathrm{g}\end{array}$} & \multicolumn{3}{|c|}{$\begin{array}{c}\text { Spleen sodium } \\
\text { concentrations } \mathrm{mg} / \mathrm{g}\end{array}$} & \multicolumn{3}{|c|}{$\begin{array}{c}\text { Brain sodium } \\
\text { concentrations } \mathbf{m g} / \mathrm{g}\end{array}$} \\
\hline $\begin{array}{c}\text { Group } \\
s(n=6 \\
)\end{array}$ & $\begin{array}{c}\text { 4wee } \\
\mathrm{k}\end{array}$ & $\begin{array}{c}8 \\
\text { week }\end{array}$ & $\begin{array}{c}12 \text { wee } \\
k\end{array}$ & $\begin{array}{c}\text { 4wee } \\
\mathrm{k}\end{array}$ & 8week & $\begin{array}{c}12 \text { wee } \\
k\end{array}$ & 4week & 8week & $\begin{array}{c}12 \text { wee } \\
k\end{array}$ & 4week & $\begin{array}{c}8 \text { wee } \\
k\end{array}$ & $\begin{array}{c}12 \text { wee } \\
k\end{array}$ \\
\hline $\begin{array}{l}\text { Contr } \\
\text { ol }\end{array}$ & $\begin{array}{c}43.25 \\
\pm 4.13 \\
\mathrm{a} \\
\end{array}$ & $\begin{array}{c}51.45 \\
\pm 3.52 \\
\text { b }\end{array}$ & $\begin{array}{c}50.06 \pm \\
2.74 \mathrm{a}\end{array}$ & $\begin{array}{c}85.47 \\
\pm 4.43 \\
\text { b } \\
\end{array}$ & $\begin{array}{c}86.69 \\
\pm 3.59 \\
\mathrm{~b} \\
\end{array}$ & $\begin{array}{c}91.57 \\
\pm 2.05 \\
\text { b }\end{array}$ & $\begin{array}{c}105.0 \\
0 \pm 4.6 \\
2 \mathrm{c} \\
\end{array}$ & $\begin{array}{c}117.5 \\
8 \pm 3.7 \\
5 \mathrm{c}\end{array}$ & $\begin{array}{l}116.68 \\
\pm 2.92 b\end{array}$ & $\begin{array}{r}39.59 \\
\pm 3.43 \mathrm{a}\end{array}$ & $\begin{array}{c}36.98 \\
\pm 2.54 \\
\mathrm{a} \\
\end{array}$ & $\begin{array}{r}36.45 \\
\pm 1.69 \mathrm{a}\end{array}$ \\
\hline $\begin{array}{l}200 \mathrm{pp} \\
\mathrm{m}\end{array}$ & $\begin{array}{c}81.54 \\
\pm 2.45 \\
\mathrm{~b}\end{array}$ & $\begin{array}{r}98.98 \\
\pm 2.76 \mathrm{c}\end{array}$ & $\begin{array}{l}104.51 \\
\pm 4.17 \mathrm{~b}\end{array}$ & $\begin{array}{c}56.69 \\
\pm 5.21 \\
\text { a }\end{array}$ & $\begin{array}{l}69.33 \\
\pm 4.37 \mathrm{a}\end{array}$ & $\begin{array}{l}74.56 \\
\pm 4.36 \mathrm{a}\end{array}$ & $\begin{array}{l}96.08 \\
\pm 3.74 \mathrm{c}\end{array}$ & $\begin{array}{c}105.3 \\
8 \pm 5.3 \\
8 \mathrm{c}\end{array}$ & $\begin{array}{l}121.08 \\
\pm 3.05 b\end{array}$ & $\begin{array}{c}45.93 \\
\pm 5.02 \mathrm{a} \\
\mathrm{b}\end{array}$ & $\begin{array}{c}64.23 \\
\pm 3.99 \\
\mathrm{~b}\end{array}$ & $\begin{array}{c}73.98 \\
\pm 4.03 \\
\mathrm{~b}\end{array}$ \\
\hline $\begin{array}{l}300 \mathrm{pp} \\
\mathrm{m}\end{array}$ & $\begin{array}{c}83.43 \\
\pm 6.85 \\
\text { b }\end{array}$ & $\begin{array}{c}109.3 \\
0 \pm 7.1 \\
1 \mathrm{c}\end{array}$ & $\begin{array}{l}117.00 \\
\pm 5.42 \mathrm{~b}\end{array}$ & $\begin{array}{c}62.65 \\
\pm 6.26 \\
\mathrm{a}\end{array}$ & $\begin{array}{c}129.2 \\
2 \pm 6.8 \\
5 c\end{array}$ & $\begin{array}{c}131.8 \\
3 \pm 5.2 \\
9 \mathrm{c}\end{array}$ & $\begin{array}{c}18.61 \\
\pm 3.29 \mathrm{a}\end{array}$ & $\begin{array}{l}21.95 \\
\pm 3.72 \mathrm{a}\end{array}$ & $\begin{array}{c}45.78 \pm \\
9.12 \mathrm{a}\end{array}$ & $\begin{array}{r}67.30 \\
\pm 4.63 \mathrm{c}\end{array}$ & $\begin{array}{c}89.83 \\
\pm 4.57 \\
\text { c }\end{array}$ & $\begin{array}{c}104.2 \\
2 \pm 6.0 \\
0 \mathrm{c}\end{array}$ \\
\hline $\begin{array}{l}400 p p \\
\quad m\end{array}$ & $\begin{array}{c}39.39 \\
\pm 9.87 \\
\text { a }\end{array}$ & $\begin{array}{r}32.39 \\
\pm 5.73 \mathrm{a}\end{array}$ & $\begin{array}{c}42.44 \pm \\
5.77 \mathrm{a}\end{array}$ & $\begin{array}{c}70.20 \\
\pm 3.87 \\
\text { a }\end{array}$ & $\begin{array}{c}132.1 \\
0 \pm 3.7 \\
7 \mathrm{c}\end{array}$ & $\begin{array}{c}122.0 \\
9 \pm 7.5 \\
1 \mathrm{c}\end{array}$ & $\begin{array}{c}47.82 \\
\pm 3.07 \\
\text { b }\end{array}$ & $\begin{array}{c}47.97 \\
\pm 5.03 \\
\text { b }\end{array}$ & $\begin{array}{c}57.10 \pm \\
4.26 \mathrm{a}\end{array}$ & $\begin{array}{c}56.83 \\
\pm 6.78 \\
\text { bc }\end{array}$ & $\begin{array}{c}62.50 \\
\pm 3.43 \\
\mathrm{~b}\end{array}$ & $\begin{array}{c}79.36 \\
\pm 3.60 \\
b\end{array}$ \\
\hline
\end{tabular}

Table 3: The Mean Sodium Concentrations in Liver, Kidney, Spleen and Brain of Male Wistar Rats Treated With Graded Doses of Lead for A Period of Four, Eight and Twelve Weeks.

Table 4 shows the liver, kidney, spleen and brain potassium concentrations of male Wistar rats treated with graded doses of lead for a period of four, eight and twelve weeks. In the liver of rats treated with graded doses of lead there was significant $(\mathrm{p}<0.05)$ reduction in the liver potassium concentration compared with the control group except for eight week exposure at 200ppm where there was significant increase in the liver potassium concentration. There was significant $(\mathrm{p}<0.05)$ increase in the kidney potassium concentration compared with the control group, however treatment for twelve weeks at $200 \mathrm{ppm}$ and $400 \mathrm{ppm}$, there was significant $(p<0.05)$ increase in kidney potassium compared with the control group. In the spleen, there was significant $(p<0.05)$ increase in the potassium concentration throughout the duration of treatment with lead, while the brain potassium concentration was significantly $(p<0.05)$ decreased compared with the control group.

\begin{tabular}{|c|c|c|c|c|c|c|c|c|c|c|c|c|}
\hline & \multicolumn{3}{|c|}{$\begin{array}{c}\text { Liver potassium } \\
\text { concentrations } \mathrm{mg} / \mathrm{g}\end{array}$} & \multicolumn{3}{|c|}{$\begin{array}{c}\text { Kidney potassium } \\
\text { concentrations mg/g }\end{array}$} & \multicolumn{3}{|c|}{$\begin{array}{c}\text { Spleen potassium } \\
\text { concentrations } \mathrm{mg} / \mathrm{g}\end{array}$} & \multicolumn{3}{|c|}{$\begin{array}{c}\text { Brain potassium } \\
\text { concentrations } \mathrm{mg} / \mathrm{g}\end{array}$} \\
\hline $\begin{array}{c}\text { Groups } \\
(\mathrm{n}=6)\end{array}$ & $\begin{array}{c}\text { 4wee } \\
\mathrm{k}\end{array}$ & $\begin{array}{c}8 \\
\text { week }\end{array}$ & $\begin{array}{c}12 \mathrm{we} \\
\mathrm{ek}\end{array}$ & 4week & $\begin{array}{c}8 w e e \\
k\end{array}$ & $\begin{array}{c}12 \mathrm{we} \\
\mathrm{ek}\end{array}$ & $\begin{array}{c}4 w e e \\
k\end{array}$ & $\begin{array}{c}8 \text { wee } \\
\mathrm{k}\end{array}$ & $\begin{array}{c}12 \mathrm{we} \\
\mathrm{ek}\end{array}$ & $\begin{array}{c}\text { 4wee } \\
\mathrm{k}\end{array}$ & 8week & $\begin{array}{c}\begin{array}{c}12 \mathrm{we} \\
\mathrm{ek}\end{array} \\
\end{array}$ \\
\hline $\begin{array}{c}\text { Contro } \\
1\end{array}$ & $\begin{array}{l}0.69 \pm \\
0.04 \mathrm{c}\end{array}$ & & & & & & $\begin{array}{l}0.67 \pm \\
0.06 \mathrm{a}\end{array}$ & & $\begin{array}{l}0.67 \pm \\
0.04 \mathrm{a}\end{array}$ & $\begin{array}{l}1.28 \pm \\
0.02 b\end{array}$ & & $\begin{array}{l}1.51 \pm \\
0.06 \mathrm{~b}\end{array}$ \\
\hline $\begin{array}{c}200 p p \\
\text { m }\end{array}$ & $\begin{array}{l}0.35 \pm \\
0.06 \mathrm{~b}\end{array}$ & & & & & & $\begin{array}{l}0.91 \pm \\
0.06 b\end{array}$ & & $\begin{array}{l}1.12 \pm \\
0.04 \mathrm{~b}\end{array}$ & $\begin{array}{l}0.95 \pm \\
0.05 c\end{array}$ & & $\begin{array}{l}1.24 \pm \\
0.03 a\end{array}$ \\
\hline $\begin{array}{c}300 \mathrm{pp} \\
\mathrm{m}\end{array}$ & $\begin{array}{l}0.37 \pm \\
0.03 \mathrm{~b}\end{array}$ & $\begin{array}{l}0.44 \pm \\
0.02 b\end{array}$ & $\begin{array}{l}0.54 \pm \\
0.04 \mathrm{~b}\end{array}$ & $\begin{array}{l}0.23 \pm 0 \\
.04 \mathrm{a}\end{array}$ & & & $\begin{array}{l}0.86 \pm \\
0.04 \mathrm{~b}\end{array}$ & & & $\begin{array}{l}1.00 \pm \\
0.06 \mathrm{a}\end{array}$ & $\begin{array}{l}1.23 \pm 0 \\
.05 b\end{array}$ & $\begin{array}{l}1.24 \pm \\
0.05 a\end{array}$ \\
\hline $\begin{array}{c}400 p p \\
\text { m }\end{array}$ & $\begin{array}{l}0.22 \pm \\
0.03 a\end{array}$ & $\begin{array}{l}0.32 \pm \\
0.03 a\end{array}$ & $\begin{array}{l}0.39 \pm \\
0.03 \mathrm{a}\end{array}$ & $\begin{array}{l}0.46 \pm 0 \\
.04 \mathrm{~b}\end{array}$ & $\begin{array}{l}0.59 \pm \\
0.04 \mathrm{~b}\end{array}$ & $\begin{array}{l}0.66 \pm \\
0.04 \mathrm{~b}\end{array}$ & $\begin{array}{l}1.00 \pm \\
0.08 \mathrm{~b}\end{array}$ & $\begin{array}{l}1.40 \pm \\
0.04 \mathrm{c}\end{array}$ & $\begin{array}{l}1.47 \pm \\
0.03 \mathrm{c}\end{array}$ & $\begin{array}{l}0.90 \pm \\
0.02 \mathrm{a}\end{array}$ & $\begin{array}{l}1.04 \pm 0 \\
.04 \mathrm{a}\end{array}$ & $\begin{array}{l}1.19 \pm \\
0.05 \mathrm{a}\end{array}$ \\
\hline
\end{tabular}

Table 4: The Mean Potassium Concentrations in Liver, Kidney, Spleen and Brain of Male Wistar Rats Treated With Graded Doses of Lead for a Period of Four, Eight and Twelve Weeks.

Values are mean \pm SEM. Values within the same column with different superscripts are significantly different at $\mathrm{p}<0.05$ 
Table 5 shows the liver, kidney, spleen and brain calcium concentrations of male Wistar rats treated with graded doses of lead for a period of four, eight and twelve weeks. There was significant $(\mathrm{p}<0.05)$ increase in the liver calcium concentration compared with the control group most especially at twelve weeks of treatment. There was significant increase $(p<0.05)$ in the kidney calcium concentration except at four weeks of treatment with the dose of 200ppm where there was significant reduction $(p<0.05)$ compared with the control. The spleen calcium concentration was also significantly $(\mathrm{p}<0.05)$ increased. There was significant reduction $(p<0.05)$ in the brain calcium concentration at eight and twelve weeks postexposure.

\begin{tabular}{|c|c|c|c|c|c|c|c|c|c|c|c|c|}
\hline & \multicolumn{3}{|c|}{$\begin{array}{c}\text { Liver calcium } \\
\text { concentration } \mathrm{mg} / \mathrm{g}\end{array}$} & \multicolumn{3}{|c|}{$\begin{array}{c}\text { Kidney calcium } \\
\text { concentration } \mathrm{mg} / \mathrm{g}\end{array}$} & \multicolumn{3}{|c|}{$\begin{array}{c}\text { Spleen calcium } \\
\text { concentration } \mathrm{mg} / \mathrm{g}\end{array}$} & \multicolumn{3}{|c|}{$\begin{array}{c}\text { Brain calcium } \\
\text { concentration } \mathrm{mg} / \mathrm{g}\end{array}$} \\
\hline $\begin{array}{l}\text { Groups } \\
(\mathrm{n}=6)\end{array}$ & 4week & $\begin{array}{c}8 \\
\text { week }\end{array}$ & $\begin{array}{c}12 \mathrm{we} \\
\mathrm{ek}\end{array}$ & 4week & 8week & $\begin{array}{c}12 \mathrm{we} \\
\mathrm{ek}\end{array}$ & 4week & 8week & $\begin{array}{c}12 \mathrm{we} \\
\mathrm{ek}\end{array}$ & 4week & 8week & $\begin{array}{c}12 \mathrm{we} \\
\mathrm{ek}\end{array}$ \\
\hline Control & $1.83 \pm$ & $1.90 \pm$ & $1.88 \pm$ & $0.33 \pm$ & $0.33 \pm$ & $0.36 \pm$ & $0.88 \pm$ & $097+$ & $0.94 \pm$ & $2.13 \pm$ & $225+$ & $2.28 \pm$ \\
\hline Contror & $0.06 \mathrm{~b}$ & $0.06 \mathrm{~b}$ & $0.06 \mathrm{~b}$ & $0.03 a$ & $0.01 \mathrm{a}$ & $0.02 \mathrm{a}$ & $0.02 \mathrm{~b}$ & 0.0 & $0.02 \mathrm{a}$ & $0.07 \mathrm{c}$ & 0.0 & $0.04 c$ \\
\hline $200 \mathrm{pp}$ & $0.88 \pm$ & $2.04 \pm$ & $2.12 \pm$ & $0.28 \pm$ & $0.96 \pm$ & $1.02 \pm$ & $0.16 \pm$ & $1.10 \pm$ & $1.16 \pm$ & $1.04 \pm$ & $1.35 \pm$ & $1.39 \pm$ \\
\hline $\mathrm{m}$ & $0.02 \mathrm{a}$ & $0.09 c$ & $0.08 c$ & $0.03 a$ & $0.03 \mathrm{~d}$ & $0.03 d$ & $0.08 \mathrm{a}$ & $0.03 b$ & $0.03 \mathrm{~b}$ & $0.08 \mathrm{a}$ & $0.07 \mathrm{~b}$ & $0.07 \mathrm{~b}$ \\
\hline $300 \mathrm{pp}$ & $1.89 \pm$ & $1.40 \pm$ & $1.88 \pm$ & $1.57 \pm$ & $0.87 \pm$ & $0.93 \pm$ & $1.46 \pm$ & & $1.68 \pm$ & $1.88 \pm$ & $1.37 \pm$ & $1.40 \pm$ \\
\hline $\mathrm{m}$ & $0.06 \mathrm{~b}$ & $0.03 a$ & $0.06 \mathrm{~b}$ & $0.69 \mathrm{~b}$ & $0.02 \mathrm{c}$ & $0.01 \mathrm{c}$ & $0.06 c$ & 0.0 & $0.04 \mathrm{c}$ & $0.08 \mathrm{~b}$ & $0.04 \mathrm{~b}$ & $0.04 \mathrm{~b}$ \\
\hline $400 \mathrm{pp}$ & $1.93 \pm$ & $1.26 \pm$ & $2.12 \pm$ & $1.54 \pm$ & $0.77 \pm$ & $0.81 \pm$ & $1.78 \pm$ & 1.1 & $1.21 \pm$ & $0.98 \pm$ & $0.91 \pm$ & $1.00 \pm$ \\
\hline $\mathrm{m}$ & $0.08 \mathrm{~b}$ & $0.06 \mathrm{c}$ & $0.08 \mathrm{c}$ & $0.06 \mathrm{~b}$ & $0.03 b$ & $0.02 b$ & $0.15 d$ & $0.02 \mathrm{~b}$ & $0.05 b$ & $0.05 \mathrm{a}$ & $0.05 a$ & $0.04 \mathrm{a}$ \\
\hline
\end{tabular}

Table 5: The Mean Calcium Concentrations in Liver, Kidney, Spleen and Brain of Male Wistar Rats Treated With Graded Doses of Lead For a Period of Four, Eight and Twelve Weeks.

Values are mean \pm SEM. Values within the same column with different superscripts are significantly different at $\mathrm{p}<0.05$

Table 6 shows the liver, kidney, spleen and brain magnesium concentrations of male Wistar rats treated with graded doses of lead for a period of four, eight and twelve weeks. For four weeks treatment, there was significant reduction in the liver magnesium concentration while for twelve weeks treatment there was an increase in the liver calcium concentration compared to the control group. The kidney magnesium concentration was statistically different from the control group. There was significant reduction at eight weeks treatment compared with the control group. The spleen magnesium concentration was significantly reduced at four weeks treatment compared with the control group while the brain magnesium concentration was slightly increased at twelve weeks exposure compared with the control group.

\begin{tabular}{|c|c|c|c|c|c|c|c|c|c|c|c|c|}
\hline & \multicolumn{3}{|c|}{$\begin{array}{c}\text { Liver Magnesium } \\
\text { concentrations mg/g }\end{array}$} & \multicolumn{3}{|c|}{$\begin{array}{l}\text { Kidney Magnesium } \\
\text { concentrations mg/g }\end{array}$} & \multicolumn{3}{|c|}{$\begin{array}{c}\text { Spleen magnesium } \\
\text { concentrations } \mathrm{mg} / \mathrm{g}\end{array}$} & \multicolumn{3}{|c|}{$\begin{array}{c}\text { Brain magnesium } \\
\text { concentrations } \mathrm{mg} / \mathrm{g}\end{array}$} \\
\hline $\begin{array}{l}\text { Groups } \\
(\mathrm{n}=6)\end{array}$ & $\begin{array}{c}\text { 4wee } \\
\mathrm{k}\end{array}$ & $\begin{array}{c}8 \\
\text { week }\end{array}$ & $\begin{array}{c}12 \text { wee } \\
\mathrm{k}\end{array}$ & $\begin{array}{c}4 \text { wee } \\
\mathrm{k}\end{array}$ & $\begin{array}{c}8 w e e \\
k\end{array}$ & $\begin{array}{c}12 \mathrm{we} \\
\text { ek }\end{array}$ & 4week & $\begin{array}{c}8 w e e \\
k\end{array}$ & $\begin{array}{c}12 \mathrm{we} \\
\text { ek }\end{array}$ & $\begin{array}{c}\text { 4wee } \\
\mathrm{k}\end{array}$ & $\begin{array}{c}8 w e e \\
k\end{array}$ & $\begin{array}{c}12 \text { wee } \\
\mathrm{k}\end{array}$ \\
\hline $\begin{array}{c}\text { Contro } \\
1\end{array}$ & & & & & & & & & & & & $\begin{array}{c}0.56 \pm 0 \\
.02 \mathrm{a}\end{array}$ \\
\hline $\begin{array}{l}200 p p \\
\text { m }\end{array}$ & $\begin{array}{l}0.10 \pm \\
0.00 \mathrm{a}\end{array}$ & & & $\begin{array}{l}0.10 \pm \\
0.00 \mathrm{a}\end{array}$ & & & $\begin{array}{l}0.44 \pm 0 \\
.04 \mathrm{ab}\end{array}$ & & & $\begin{array}{l}0.11 \pm \\
0.01 \mathrm{a}\end{array}$ & & $\begin{array}{l}0.62 \pm 0 \\
.03 \mathrm{ab}\end{array}$ \\
\hline $\begin{array}{c}300 \mathrm{pp} \\
\mathrm{m}\end{array}$ & $\begin{array}{l}0.10 \pm \\
0.00 \mathrm{a}\end{array}$ & & $\begin{array}{l}0.66 \pm 0 \\
.02 \mathrm{ab}\end{array}$ & $\begin{array}{l}0.10 \pm \\
0.00 \mathrm{a}\end{array}$ & & & $\begin{array}{l}0.34 \pm 0 \\
.05 \mathrm{a} \\
\end{array}$ & & & $\begin{array}{l}0.56 \pm \\
0.05 \mathrm{~b}\end{array}$ & & $\begin{array}{l}0.69 \pm 0 \\
.01 \mathrm{~b} \\
\end{array}$ \\
\hline $\begin{array}{c}400 \mathrm{pp} \\
\mathrm{m}\end{array}$ & $\begin{array}{l}0.10 \pm \\
0.00 \mathrm{a}\end{array}$ & $\begin{array}{l}0.66 \pm \\
0.01 \mathrm{a}\end{array}$ & $\begin{array}{l}0.69 \pm 0 \\
.02 \mathrm{~b} \\
\end{array}$ & $\begin{array}{l}0.10 \pm \\
0.00 \mathrm{a}\end{array}$ & $\begin{array}{l}0.51 \pm \\
0.03 \mathrm{c}\end{array}$ & $\begin{array}{l}0.63 \pm \\
0.03 \mathrm{a}\end{array}$ & $\begin{array}{l}0.55 \pm 0 \\
.03 \mathrm{bc} \\
\end{array}$ & $\begin{array}{l}0.61 \pm \\
0.05 \mathrm{a}\end{array}$ & $\begin{array}{l}0.64 \pm \\
0.03 \mathrm{a}\end{array}$ & $\begin{array}{l}0.57 \pm \\
0.09 \mathrm{~b}\end{array}$ & $\begin{array}{l}0.63 \pm \\
0.05 \mathrm{a}\end{array}$ & $\begin{array}{l}0.62 \pm 0 \\
.05 \mathrm{ab}\end{array}$ \\
\hline
\end{tabular}

Table 6: The Mean Magnesium Concentrations in Liver, Kidney, Spleen and Brain of Male Wistar Rats Treated with Graded Doses of Lead for a Period of Four, Eight and Twelve Weeks.

Values are mean \pm SEM. Values within the same column with different superscripts are significantly different at $\mathrm{p}<0.05$ 


\section{Advances in Clinical Toxicology}

\section{Discussion}

Lead is a ubiquitous and versatile metal which has been used by mankind for many years. It ranks as one of the most serious environmental poisons amongst the toxic heavy metals all over the world. Exposure to lead is from numerous sources and a myriad of pathways including air, food, dust, soil and water [3]. Accumulation of lead produces damaging effects in the hematopoetical, hematic, renal and gastrointestinal systems [4]. The result of this study shows accumulation of lead in the blood upon absorption, this is due to affinity of lead for red blood cells which transport them to various parts of the body and soft tissues where they are stored and results in perturbation of metabolic processes. Our results is consistent with other workers Warren, et al. [4], Murata, et al. and Okediran, et al. reported blood lead accumulation following oral exposure resulting in loss of weight, inhibition of haem synthesis and reduction of packed cell volume $[16,17]$.

Electrolytes plays significant role in several body processes, such as controlling fluid levels, acid-base balance $(\mathrm{pH})$, nerve conduction, coagulation and muscle contraction [18]. Fluid and electrolytes homeostasis is usually maintained within narrow limits [19] and therefore, it must be kept at a level that is suitable for normal biochemical and physiological functions [20]. Therefore, imbalance in serum electrolytes is an important indicator of toxicity in identifying target organs and general health status of animals. Decrease in plasma electrolytes concentrations of sodium, potassium, calcium and magnesium was observed in this study, it is an indication in perturbation of electrolytes metabolism due to exposure to lead thus leading to alterations in cellular functions in which these electrolytes are involved. It was reported that lead can replace calcium, thereby affecting key neurotransmitters like protein kinase $C$, which regulates long term neural excitation and memory storage, it also affect sodium ion concentration which is responsible for numerous vital biological activities like generation of action potentials in the excitatory tissues for the purpose of cell to cell communication, uptake of neurotransmitters and regulation of uptake and retention of calcium by synaptosomes [2,21-23].

The ionic mechanism of action for lead mainly arises due to its ability to substitute other bivalent cations such calcium, magnesium, iron and monovalent cations like sodium ions, thus affecting various fundamental biological processes of the body by damaging cellular components $[24,25]$. Lead concentration in the blood is transient and only represents recent exposure of some days, as blood flows through the soft tissues, lead is deposited and bioaccumulated [26].

Exposure to lead mainly occurs through the respiratory and gastrointestinal systems. Absorbed lead is conjugated in the liver and passed to the kidney, where a small quantity is excreted in urine and the rest accumulates in various body organs. This affects many biological activities at the molecular, cellular and intercellular levels, which may result in morphological alterations that can remain even after lead level has fallen [7-9,27].

Autopsy studies of lead exposed animals indicated that liver tissue is the largest repository of lead among soft tissues followed by kidney cortex and medulla. Lead induced hepatotoxicity was reported to be associated with the impairments of liver structure and function [28]. Environmental exposures to lead have caused nephrotoxicity in humans and animals [29]. Bio makers of kidney functions have been reported to be impaired by lead in humans and animals [30-32].

In the brain exposure of animals to lead caused impairments in hippocampus, cerebellum, cerebellar edema, cerebral satellitosis and encephalomalacia [33]. The liver is the major organ of drug metabolism and is highly exposed to both indigenous and exogenous chemical substances. Studies have shown that the liver is one of the primary targets in lead associated toxicity. Metabolism in the liver usually leads to detoxification of environmental compounds; hence the concept that the liver purifies or cleanses the blood [34]. The kidney is the major organ of drug excretion and is also one of the organs of primary target during lead toxicity. There are reports from some quarters on lead induced kidney damage in experimental animal studies and humans [35].

They brain is an essential organ of the body which is reported to be impaired on exposure to lead with respect to time. Xu, et al. [36] reported that exposure to lead before and after birth can damage short-term and longterm memory ability of young rats and hippocampal ultrastructure. The spleen plays a major role in the destruction of effete red cell thereby removing and storage of iron. The iron stored is readily released for haematopoietic.

In all these organs, ATPases play important roles in intracellular functions and for all types of physiological activities. It has been reported that inhibition of ATPases activities is used as a sensitive indicator of heavy metal toxicity in red cells, rat brain cell and rat liver cells $[37,38]$, while lead compound can enhance per oxidative damage 


\section{Advances in Clinical Toxicology}

to the membranes of blood cells, liver cells and other cells thus compromising cellular functions $[39,40]$.

In all these organs in this study, lead was reported to cause alterations in the electrolytes in these organs. It was reported that one of the potential mechanisms by which lead exhibit it toxicity is by inhibition of ATPases which is a membrane bound enzymes. It could be that inhibition of these ATPases activities which is responsible for electrolytes movement was overwhelmed and thus result in alterations of electrolytes in these organs studied. Also since membrane is made up of lipids there is every possibility that lead alters the membrane structure and could trigger lipid peroxidation thus disturbing the ATPases conformation and configuration and hence distort the electrolytes movement. Carafoli [41] reported inhibition of $\mathrm{Ca}^{2+}-\mathrm{Mg}^{2+}$ ATPases activities, while Guzide, et al. [42] reported inhibition of $\mathrm{Na}^{+}-\mathrm{K}^{+}$ATPase and $\mathrm{Ca}^{2+}$ $\mathrm{Mg}^{2+}$ ATPases activities, transport proteins, which regulate cations flux involved in maintaining and regulating intracellular cation content by lead. Bettaiya, et al. [37] showed that lead chloride and lead acetate at micromolar concentrations significantly inhibited the $\mathrm{Ca}^{2+}$ ATPase at cellular membranes and microsomes of cerebral cortex and cerebellum in a concentrationdependent manner.

The important implication of this study is that exposure to lead is equally responsible for alteration in the electrolytes distributions in the blood as well as in organs and tissues. These alterations in these electrolytes results in impairment of physiological and metabolic activities taken place in these organs.

Our results indicate that one of the ways that lead exerts its toxic effects is disruption of electrolytes balance thus deteriorating cellular functions.

\section{References}

1. Shalan MG, Mostafa MS, Hassouna MM, Nabi SE, Rafie A (2005) Amelioration of lead toxicity on rat liver with vitamin $C$ and silymarin supplements. Toxicol 206(1): 1-15.

2. Garaza A, Vega R, Soto E (2006) Cellular mechanisms of lead neurotoxicity. Med Sci Mon 12(3): 57-65.

3. Herman DS, Geraldine M, Venkatesh T (2007) Case report: Evaluation, diagnosis, and treatment of lead poisoning in a patient with occupational lead exposure: a case presentation. J Occup Med Toxicol 2: 7-10.
4. Correia PRM, Oliveira E, Oliveira PV (2000) Simultaneous determination of $\mathrm{Cd}$ and $\mathrm{Pb}$ in foodstuffs by electrothermal atomic absorption spectrometry. Anal Acta 405(1-2): 205-211.

5. Roh YH, Kim K, Kim H (2000) Zinc protoporphyrin IX concentrations between normal adults and the leadexposed workers measured by HPLC, spectrofluorometer, and hematofluorometer. Ind Hlth 38(4): 372-379.

6. Garrettson LK (1990) Lead in Clinical Management of poisoning and drug over-dose. In: Haddad LM, et al. (Eds.), Philadelphia: W Saunders Company, pp: 101723.

7. Sidhu P, Nehru B (2004) Lead intoxication: Histological and oxidative damage in rat cerebrum and cerebellum. J Trace Elem Exp Med 17(1): 45-53.

8. Taib NT, Jarrar BM, Mubarak M (2004) Ultrastructural alterations in hepatic tissues of white rats (Rattus norvegicus) induced by lead experimental toxicity. Saudi J Biol Sci 11(1): 11-20

9. Flora SJS, Flora G, Saxena G (2006) Environmental Occurrence, Health Effects and Management of Lead Poisoning. In: Casas SB (Eds.), Lead: Chemistry, Analytical Aspects, Environmental Impact and Health Effects. Elsevier, Nethlands, pp: 158-228.

10. Berrahal AA, Lasram M, El-Elj N, Kerkeni A, Gharbi N, et al. (2011) Effect of age-dependent exposure to lead on hepatotoxicity and nephrotoxicity in male rats. J Environ Toxicol 26(1): 68-78.

11. Johny CP, Oscar PM, Rolando HM, Patricia VT, Marco AJ (2010) Protective effects of Spirulina maxima on hyperlipidemia and oxidative-stress induced by lead acetate in the liver and kidney. Lipids in health and Dis 9: $35-41$.

12. Trinder P (1951) Quantitive determination of serum sodium by colorimetric method. Anal 76: 596-98.

13. Terri AE, Sesin PG (1958) Quantitative determination of serum potassium by nephelometric method. Ame J Clin Pathol 29: 86-88.

14. Schmidt-Gayk H, Blind E, Roth HJ (1953) Hormones and markers of bone metabolism. Measurement and interpretation, $2^{\text {nd }} \quad($ Edn.), Clinical Laboratory Publications, Heidelberg. 


\section{Advances in Clinical Toxicology}

15. Abernethy MH, Fowler RT (1982) Micellar improvement of the calmagite compleximetric measurement of magnesium in plasma. Clin Chem 28(3): 520-22.

16. Murata K, Sakai T, Morita Y, Iwata T, Dakeishi M (2003) Critical dose of lead affecting -Amino Levunic Acid levels. J Occup Hth 45: 209-214.

17. Okediran BS, Kasali OB, Omotainse SO, Akinloye OA (2016) Haemato-biochemical alterations as Biomarkers of lead induced toxicity in male Wistar rats. Bang J Vet Med 14(2): 227-232.

18. Hasona NA, Elasbali A (2016) Evaluation of electrolytes imbalance and dyslipidaemia in diabetic patients. Med Sci 4(2): 1-4.

19. Roberts KE (2005) Pediatric fluid and Electrolytes Balance: Critical Care Case Studies. Crit Care Nurs Clin Nor Ame 17(4): 361-373.

20. Kaneko JJ, Harvey JW, Bruss ML (2008) Clinical Biochemistry of Domestic Animals, $6^{\text {th }}$ (Edn.). Academic, London, pp:117-38

21. Bressler J, Kim KA, Chakraborti T, Goldstein G (1999) Molecular mechanisms of lead neurotoxicity. Neurochem Res 24(4): 595-600.

22. Gwaltney-Brant SM (2002) Heavy Metals, Handbook of Toxicologic Pathology. $2^{\text {nd }}$ (Edn.), In: Haschek WM, et al. (Eds.), Academic Press, pp: 701-733.

23. Hontela A, Lacroix A (2006) Heavy Metals in endocrine disruptors: Biological Basis for Health effects in Wildlife and Human. In: Carr J, et al. (Eds.), Oxford University Press, United Kingdom, pp: 356374.

24. Foyer $\mathrm{CH}$, Noctor G (2000) Oxygen processing in photosynthesis: regulation and signaling. New J Phytol 146(3): 359-388.

25. Lidsky TI, Schneider JS (2003) Lead neurotoxicity in children: Basic mechanisms and clinical correlates. Brain 126(1): 5-19.

26. Babalola 00, Okonji RE, Atoyebi JO, Sennuga TF, Raimi MM, et al. (2010) Distribution of lead in selected organs and tissues of albino rats exposed to acute lead toxicity. Sci Res Ess 5(9): 845-848.
27. Ibrahim NM, Eweis EA, El-Beltagi HS, Yasmin E (2012) Effect of lead acetate toxicity on experimental male albino rat. Asian Pac J Trop. Biomed 2(1): 41-46.

28. Aziz FM, Maulood IM, Chawsheen MAH (2012) Effects of melatonin, vitamin $\mathrm{C}$ and $\mathrm{E}$ alone or in combination on lead-induced injury in liver and kidney organs of rats. IOSR J Pharm 2(5): 13-18.

29. Diamond GL (2005) Risk Assessment of Nephrotoxic Metals. In: Tarloff, J, et al. (Eds.), the Toxicology of the Kidney. CRC Press, London, pp: 1099-1132.

30. Gurer-Orhan H, Sabir HU, Ozgune H (2004) Correlation between clinical indicators of lead poisoning and xidative stress parameters in controls and lead-exposed workers. Toxicol 195(2-3): 147154.

31. Weaver VM, Jaar BG, Schwartz BS, Todd AC (2005) Associations among lead dose biomarkers, uric acid and renal function in Korean leadworkers. Environ Health Perspect 113(1): 36-42.

32. Ahmed K, Ayana G, Engidawork E (2008) Lead exposure study among workers in lead acid battery repair units of transport service enterprises, Addis Ababa, Ethiopia: A cross-sectional study. J Occupat Med Toxicol 3(30): 1-8.

33. El-Neweshy M, El-Sayed, Y (2011) Influence of vitamin $\mathrm{C}$ supplementation on lead-induced histopathological alterations in male rats. Exp Toxicol Pathol 63(3): 221-227.

34. Howard JW (1999) The liver disorders sourcebook Lowell House, Los Angeles, pp: 5.

35. Missoun F, Slimani M, Aoues, A (2010) Toxic effect of lead on kidney function in Wistar rat. Afri J Biochem Res 4(2): 21-27.

36. Xu J, Yan HC, Yang B, Tong LS, Zou YX (2009) Effects of lead exposure on hippocampal metabotropic glutamate receptor subtype 3 and 7 in developmental rats. J Negative Results BioMed 8(5): 1-8.

37. Bettaiya R, Yallapragada, PR, Hall E, Rajanna S (1996) In vitro effect of lead on $\mathrm{Ca}^{2+}$ - ATPase in synaptic plasma membrane and microsomes of rat cerebral cortex and cerebellum. Exotoxicol Environ Safety 33(2): 157-162.

38. Prabhakara Rao JS, Rajanna SF, Rajanna B (1996) Inhibition of calcium transport by mercury salts in rat 
cerebellum and cerebral cortex in vitro. J Applied Toxicol 16(4): 325-330.

39. Samarghandian S, Borji A, Afshari R, Delkhosh MB, Gholami A (2013) The effect of lead acetate on oxidative stress and antioxidant status in rat bronchoalveolar lavage fluid and lung tissue. Toxicol Mech Methods 23(6): 432-436.

40. Patra RC, Swarup D, Dwivedi SK (2001) Antioxidant effects of $\alpha$ tocopherol, ascorbic acid and L- methionine on lead induced oxidative stress to the liver, kidney and brain in rats. Toxicol 162(2): 81-88.

41. Carafoli E (1987) Intracellular calcium homeostasis. Ann Rev of Biochem 56: 395-433.

42. Guzide $Y$, Ramazan R, Lulufer T, Seyham T (2003) Effects of lead on $\mathrm{Na}^{+-} \mathrm{K}^{+}$- ATPase and $\mathrm{Ca}^{2+}$-ATPase activities and lipid peroxidation in blood of workers. Inter J Toxicol 22(2): 95-97. 